which afforded $\alpha$-cyanobenzyl benzene sulphonate ${ }^{2}$, this addition compound could be formed thus: $R-\mathrm{CHO}+R^{\prime}-\mathrm{SO}_{2} \mathrm{OH} \rightleftharpoons R-\overbrace{\mathrm{H}}^{\mathrm{OH}}-\mathrm{O}-\mathrm{SO}_{2} R^{\prime}$

Another factor influencing the absorption of the aldehydes could be van der Waals adsorption forces, since it is known that compounds containing aromatic residues are adsorbed on polystyrene ion-exchange resins ${ }^{3-5}$. 'Amberlite IR $120\left(\mathrm{H}^{+}\right)^{\prime}$, which is equivalent to 'Zeo-Karb' $225\left[\mathrm{H}^{+}\right]^{\text {', has been used }}$ ' to separate phenol, $p$-cresol and 4-tert. butyl phenol. Gabrielson and Samuelson ${ }^{7}$ have investigated the adsorption of aldehydes and ketones on 'Amberlite IRA-400' (bicarbonate form), which is an anion exchanger of the strongly basic type. They reported that salicylaldehyde and vanillin were strongly absorbed, whereas formaldehyde, acetaldehyde and 2 -furaldehyde were eluted almost quantitatively. In this case absorption was attributed to the acidic character of the two aromatic aldehydes.

The unexpected property of derivatives of 2 . furaldehyde may prove useful not only in the identification of intermediates in the browning reaction but also those products of acidic degradation of sugars which form the basis of so many colour reactions for the quantitative determination of sugars.

One of us (K. M.) thanks Glaxo, Ltd., for the award of a scholarship.

Chemistry Department,

The University,

Birmingham, 15.

' Hodge, J. E., J. Agric. Food Chem., 1, 928 (1953).

${ }^{2}$ Dodson, R. M., and Turner, H. N., J. Amer. Chem. Soc., Yะ, 4517 (1951).

${ }^{3}$ Partridge, S. M., Biochem. J., 44, 521; 45, 459 (1949).

'Weiss, D. E., Nature, 186, 66 (1950).

${ }^{5}$ Davies, C. W., Biochem. J., 45, 38 (1949).

'Seki, T., J. Chem. Soc. Japan, Pure Chem. Sect., 75, (12), 1297 (1954).

־Gabrielson, G., and Samuelson, O., Acta Chem. Scand., 6, 729 (1952)

\section{Symmetry of Insulin Dimers and Hexamers}

Recent X-ray crystallographic studies of orthorhombic crystals of bovine insulin sulphate have provided information about the symmetry of the insulin dimer (molecular weight $2 \times 5733$ ). This dimer appears to be the stable unit in acid aqueous solution, except under special conditions. The dimer is the protein component of the asymmetrical unit in the wet, type $A$ and type $B$ sulphate and type $A$ citrate, crystals ${ }^{2}$, and also in the air-dried sulphate erystals ${ }^{3}$. Studies of the shrinkage stages of the type $A$ insulin sulphate crystals ${ }^{4}$ have disclosed the existence of several new crystal forms. The space group of all but on $\theta$ of these is $P 2_{1} 2_{1} 2_{1} \quad(Z=4)$, that of all previously examined orthorhombic insulin crystals. One shrinkage stage (type $C$ ) has the space group $I 2_{1} 2_{1} 2_{1}(Z=8)$. In this crystal twofold rotation axes relate pairs of insulin molecules. On the basis of crystallographic and other evidence, it was possible to establish the strong probability that the insulin dimer has basically an invariant structure, with point group 2, in all these crystal forms and in acid solution; that is, the two insulin molecules of the dimer are related to each other by a twofold rotation axis.

This conclusion was strengthened by Patterson studies of the wet type $A$ insulin sulphate crystals ${ }^{5}$. The position of the highest non-origin peak in the three-dimensional Patterson function is that of a pseudo-origin in each of two Patterson projectionsthat is, the distributions of peaks about this position are similar to those about the origins of these projections. However, the pseudo-origin character appears slight or non-existent in the third ( $a$-plane) projection and in the three-dimensional Patterson function. These observations have been explained on the basis of a non-crystallographic twofold (or pseudo-twofold) rotation axis parallel (or nearly parallel) to $a$. Given two independent sources of evidence, the symmetry, or, if the dimer structure is not invariant, at least the pseudo-symmetry, of the insulin dimer seems to be established.

The predominant insulin species in solution near neutral $p \mathrm{H}$ is the hexamer ${ }^{1 b}$. A threefold rotation axis relating three dimer units has been found in rhombohedral zinc insulin crystals, space group $R 3$, grown under these $p \mathrm{H}$ conditions ${ }^{6}$. It appears to us that the dimer as a subunit of the hexamer may well have the point group 2. Indeed, it is quite possible that the hexamer has the point group 32 with each single molecule equivalent. (If the hexamer has the point group 32, then the three twofold rotation axes relating the molecules to each other in the dimer subunits lie in a plane normal to the threefold axis and at angles of $120^{\circ}$ to each other.) If non-crystallographic twofold axes should exist in the rhombohedral zinc insulin crystal form, they should be detectable, with greater or lesser ease dependent upon their orientation to the threefold axis, by analysis of the vector structure.

This investigation was supported by senior research fellowship $S F-246 C$ from the Public Health Service to one of us (B. W. L.). It was carried out during the tenure of a National Science Foundation post-doctoral fellowship (J. R. E.).

\section{Barbara W. Low}

J. RATPH EINSTEIN

Department of Biochemistry,

College of Physicians and Surgeons, Columbia University,

New York 32.

${ }^{1}$ (a) Moody," L. S., Ph.D. Dissertation, Univ. of Wisconsin (1944), quoted by Williams, J. W., "Ann. Rev. Phys. Chem.", 2, 412 (1951). (b) Oncley, J. L., Ellenbogen, E., Gitlin, D., and Gurd, F. R. N., J. Phys. Chem., 56, 85 (1952). (c) Kupke, D. W., and LinderstromLang, K., Biochim. Biophys. Acta, 13, 153 (1954). Low, B. W., and Shoemaker, C. B., Acta Cryst., 12, 893 (1959),
Low, B. W., and Berger, J. E. (in preparation).

${ }^{3}$ Low, B. W., Nature, 169,955 (1952).

4Einstein, J. R., Ph.D. thesis, Harvard University (1958). Einstein, $J$, $R$, and Low, B. W. (in preparation).

5 Shoemaker, C. B., Einstein, J. R., and Low, B. W. (in preparation). - Crowfoot, D., and Riley, D., Nature, 144, 1011 (1939).

\section{BIOCHEMISTRY}

\section{Dolichol : a Naturally Occurring Isoprenoid Alcohol}

IN $1956^{1}$ a long-chain isoprenoid alcohol, solenesol, was isolated from tobacco and tobacco leaves. While searching for solanesol in animal tissues we have isolated an isoprenoid alcohol of greater chain-length for which we propose the name 'dolichol'. 